PROCEEDINGS OF THE

AMERICAN MATHEMATICAL SOCIETY

Volume 140, Number 3, March 2012, Pages 971-975

S 0002-9939(2011)10986-9

Article electronically published on July 22,2011

\title{
SIMULTANEOUS EXTENSIONS \\ OF TURKEVICH'S INEQUALITY AND THE WEIGHTED AM-GM INEQUALITY
}

\author{
GÉZA KÓS, HOJOO LEE, AND PETER VANDENDRIESSCHE
}

(Communicated by Walter Van Assche)

ABStract. We establish a sharp homogeneous inequality which extends both the classical weighted AM-GM inequality and the Turkevich inequality.

\section{Introduction AND MAin RESUlts}

Turkevich [1] discovered a neat 4-variable symmetric inequality of degree 4:

$$
a^{4}+b^{4}+c^{4}+d^{4}+2 a b c d \geq a^{2} b^{2}+a^{2} c^{2}+a^{2} d^{2}+b^{2} c^{2}+b^{2} d^{2}+c^{2} d^{2}
$$

or

$$
\left(a^{2}-b^{2}\right)^{2}+\left(c^{2}-d^{2}\right)^{2} \geq\left(a^{2}+b^{2}\right)\left(c^{2}+d^{2}\right)-(a b+c d)^{2}
$$

for all non-negative real numbers $a, b, c, d$. Equality occurs if and only if either $a=b=c=d$ or if three of $a, b, c, d$ are equal and the remaining one is zero.

Several generalizations of Turkevich's inequality are known; for example, Shleifer's inequality [1] says that, for $a_{1}, \ldots, a_{n} \geq 0$,

$$
(n-1) \sum_{i=1}^{n} a_{i}^{4}+n\left(a_{1} \cdots a_{n}\right)^{\frac{4}{n}} \geq\left(\sum_{i=1}^{n} a_{n}^{2}\right)^{2} .
$$

The main aim of this paper is to present a sharp weighted generalization of the AM-GM inequality, which also generalizes Turkevich's inequality.

In the following, let $n$ be a positive integer with $n \geq 2$ and let $\omega_{1}, \ldots, \omega_{n}$ be positive real numbers with $\omega_{1}+\cdots+\omega_{n}=1$. Define $\omega=\min \left\{\omega_{1}, \ldots, \omega_{n}\right\}>0$ and denote $\lambda=(1-\omega)^{-\frac{1-\omega}{\omega}}>1$.

We now present our two main theorems, which will turn out to be equivalent.

Received by the editors September 26, 2010 and, in revised form, November 17, 2010 and December 18, 2010.

2010 Mathematics Subject Classification. Primary 26D15; Secondary 41A44.

Key words and phrases. Turkevich's inequality, Euler's constant, weighted means.

The second author is supported in part by NRF 2010-0014315.

The third author is supported by a PhD fellowship of the Research Foundation - Flanders (FWO). 
Theorem 1. Let $a_{1}, \ldots, a_{n}, b_{1}, \ldots, b_{n}$ be non-negative real numbers $(n \geq 2)$ and let $\omega_{1}, \ldots, \omega_{n}$ be positive weights with $\omega_{1}+\cdots+\omega_{n}=1$. We have

$$
\lambda \sum_{k=1}^{n} \omega_{k}\left(a_{k}^{2}-b_{k}^{2}\right)^{2}+\left(2 \sum_{k=1}^{n} \omega_{k} a_{k} b_{k}\right)^{2} \geq\left(a_{1}^{2}+b_{1}^{2}\right)^{2 \omega_{1}} \cdots\left(a_{n}^{2}+b_{n}^{2}\right)^{2 \omega_{n}} .
$$

Equality in (1.1) occurs if and only if we have either $a_{1}=\cdots=a_{n}=b_{1}=\cdots=$ $b_{n}$, or if we have

$$
\left|a_{k}^{2}-b_{k}^{2}\right|=\left\{\begin{array}{ll}
a & \text { if } k=i_{0} \\
0 & \text { if } k \neq i_{0}
\end{array} \text { and } 2 a_{k} b_{k}= \begin{cases}0 & \text { if } k=i_{0} \\
b & \text { if } k \neq i_{0}\end{cases}\right.
$$

for some integer $i_{0} \in\{1, \ldots, n\}$ with $\omega_{i_{0}}=\omega$ and for some $a, b \geq 0$ for which $\lambda a^{2}=b^{2}(1-\omega)$.

The existence of the equality condition guarantees the minimality of the optimal coefficient $\lambda$ in inequality (1.1). Theorem 1 is an $n$-variable generalization of Turkevich's inequality [1]; the original inequality of Turkevich can be obtained by letting $n=2$ and $\omega_{1}=\omega_{2}=\frac{1}{2}$, in which case $\lambda=2$.

To establish Theorem 1, we will use the following theorem, which is a nonsymmetric equivalent to Theorem 1 .

Theorem 2. Let $a_{1}, \ldots, a_{n}, b_{1}, \ldots, b_{n}$ be non-negative real numbers $(n \geq 2)$ and let $\omega_{1}, \ldots, \omega_{n}$ be positive weights with $\omega_{1}+\cdots+\omega_{n}=1$. Then we have

$$
\lambda \sum_{k=1}^{n} \omega_{k} a_{k}^{2}+\left(\sum_{k=1}^{n} \omega_{k} b_{k}\right)^{2} \geq\left(a_{1}^{2}+b_{1}^{2}\right)^{\omega_{1}} \cdots\left(a_{n}^{2}+b_{n}^{2}\right)^{\omega_{n}} .
$$

Equality in (1.2) occurs if and only if we either have $a_{1}=\cdots=a_{n}=0$ and $b_{1}=\cdots=b_{n}$ or we have

$$
a_{k}=\left\{\begin{array}{ll}
a & \text { if } k=i_{0} \\
0 & \text { if } k \neq i_{0}
\end{array} \text { and } b_{k}= \begin{cases}0 & \text { if } k=i_{0} \\
b & \text { if } k \neq i_{0}\end{cases}\right.
$$

for some integer $i_{0} \in\{1, \ldots, n\}$ with $\omega_{i_{0}}=\omega$ and for some $a, b \geq 0$ for which $\lambda a^{2}=b^{2}(1-\omega)$.

Inequality (1.2) is clearly a generalization of the weighted AM-GM inequality, as can be seen by substituting $a_{1}=\ldots=a_{n}=0$. That it is a strict generalization, can be seen from the additional equality conditions, where $a_{1}=\ldots=a_{n}=0$ does not necessarily hold.

Several specific estimations on the optimal coefficient $\lambda$ in Theorems 1 and 2 can be made. First, as the following proposition shows, both inequalities (1.1) and (1.2) still hold when replacing $\lambda$ with Euler's constant $e$.

Proposition 3. Let $n \geq 2$. We have $e>\lambda$ for any positive weights $\omega_{1}, \ldots, \omega_{n}$ with $\omega_{1}+\cdots+\omega_{n}=1$.

Second, the following proposition indicates that the resulting inequalities are still sharp, in the sense that $e$ cannot be replaced by a smaller constant.

Proposition 4. Let $n \geq 2$. Suppose that $\mathcal{C}$ is a positive real constant for which

$$
\mathcal{C} \sum_{k=1}^{n} \omega_{k} a_{k}^{2}+\left(\sum_{k=1}^{n} \omega_{k} b_{k}\right)^{2} \geq\left(a_{1}^{2}+b_{1}^{2}\right)^{\omega_{1}} \cdots\left(a_{n}^{2}+b_{n}^{2}\right)^{\omega_{n}}
$$


holds for all positive weights $\omega_{1}, \ldots, \omega_{n}$ with $\omega_{1}+\cdots+\omega_{n}=1$ and for all nonnegative real numbers $a_{1}, \ldots, a_{n}, b_{1}, \ldots, b_{n}$. Then $\mathcal{C} \geq e$.

If $\omega_{1}=\ldots=\omega_{n}=\frac{1}{n}$, we have $\lambda=\left(1+\frac{1}{n-1}\right)^{n-1}$. This gives our inequalities simple forms for the uniform weight distribution $\omega_{1}=\ldots=\omega_{n}=\frac{1}{n}$, and it is sharper than replacing $\lambda=\left(1+\frac{1}{n-1}\right)^{n-1}$ by Euler's constant $e$.

Theorems 1 and 2 are the main theorems of this paper. In Section 2, we present a proof of our main theorems, as well as a proof for the propositions above.

\section{Proof of the MAIN THEOREMS AND THE PRopositions}

In this section we give the proof of our main theorems. First we introduce a useful notation and we present an observation on the minimal optimal coefficient $\lambda$. Given a proper subset $I$ of $\{1, \ldots, n\}$, we denote

$$
\lambda_{I}=\left(\sum_{i \notin I} \omega_{i}\right)^{-\frac{\sum_{i \notin I} \omega_{i}}{\sum_{i \in I} \omega_{i}}}=f\left(\sum_{i \in I} \omega_{i}\right),
$$

where we define $f(x)=(1-x)^{-\frac{1-x}{x}}$. We then recall the definitions in Section 1:

$$
\omega=\min \left\{\omega_{1}, \ldots, \omega_{n}\right\}>0 \text { and } \lambda=f(\omega)=(1-\omega)^{-\frac{1-\omega}{\omega}}>1 .
$$

Since the function $f$ is decreasing on ]0,1[, we have that $\lambda_{I} \leq \lambda$ for each non-empty proper subset $I \subset\{1, \ldots, n\}$. In particular, because the function $f$ is decreasing,

$$
\lambda=\max \left\{\lambda_{I} \mid I \text { is a non-empty proper subset of }\{1, \ldots, n\}\right\}
$$

and this maximum is attained when $\sum_{i \in I} \omega_{i}$ is minimal, i.e. when $I=\left\{i_{0}\right\}$, where $i_{0}$ is any index for which $\omega_{i_{0}}=\omega$. This maximality of the minimal optimal coefficient $\lambda=f(\omega)$ is crucial to the proof of Theorem 2, We start by proving Theorem 2,

Proof of Theorem 2. Let $p_{i}=\sqrt{a_{i}^{2}+b_{i}^{2}}$ for all integers $i$, with $1 \leq i \leq n$. If there is any integer $i$, with $1 \leq i \leq n$, for which $p_{i}=0$, then the right hand side equals 0 and the inequality holds trivially. In this case equality occurs if and only if $a_{1}=\ldots=a_{n}=b_{1}=\ldots=b_{n}=0$.

Hence we may assume that $p_{i}>0$ for all integers $i, 1 \leq i \leq n$. We can rewrite the claimed estimation as

$$
\lambda \sum_{k=1}^{n} \omega_{k}\left(p_{k}^{2}-b_{k}^{2}\right)+\left(\sum_{k=1}^{n} \omega_{k} b_{k}\right)^{2} \geq p_{1}^{2 \omega_{1}} \cdots p_{n}^{2 \omega_{n}} .
$$

If we now fix the variables $p_{1}, \ldots, p_{n}, b_{1}, \ldots, b_{i-1}$ and $b_{i+1}, \ldots, b_{n}$, for some integer $i$, with $1 \leq i \leq n$, then we find that the right hand side is a constant, while the left hand side is a quadratic function of $b_{i}$ with leading coefficient $\omega_{i}\left(\omega_{i}-\lambda\right)$. Since $\lambda>1>\omega_{i}>0$, this leading coefficient is negative; thus the left hand side is a concave function in the variable $b_{i}$. Therefore, the smallest value of the left hand side is attained either when $b_{i}=0$ or $b_{i}=p_{i}$. Since this holds for any integer $i$, with $1 \leq i \leq n$, we may assume that $b_{i} \in\left\{0, p_{i}\right\}$ for each integer $i$, with $1 \leq i \leq n$.

Let $m$ be the number of integers $i$, with $1 \leq i \leq n$, for which $b_{i}=0$. We may permute the indices such that $b_{1}=b_{2}=\ldots=b_{m}=0$ and $b_{m+1}=p_{m+1}>$ $0, \ldots, b_{n}=p_{n}>0$; we denote this permutation by $\sigma$. With these observations, it is 
sufficient to prove the following inequality for arbitrary positive weights $\omega_{1}, \ldots, \omega_{n}$ with $\omega_{1}+\cdots+\omega_{n}=1$ and arbitrary positive reals $p_{1}, \ldots, p_{n}$ :

$$
\lambda \sum_{k=1}^{m} \omega_{k} p_{k}^{2}+\left(\sum_{k=m+1}^{n} \omega_{k} p_{k}\right)^{2} \geq p_{1}^{2 \omega_{1}} \cdots p_{n}^{2 \omega_{n}} .
$$

Now there are three cases: either $m=0, m=n$, or $1 \leq m \leq n-1$. If $m=0$, then (2.1) is simply the AM-GM inequality for $p_{1}, \ldots, p_{n}$. Equality hence occurs if and only if $p_{1}=\ldots=p_{n}$, which in the original problem can be written as $a_{1}=\ldots=a_{n}=0$ and $b_{1}=\ldots=b_{n}$.

If $m=n$, then

$$
\lambda \sum_{k=1}^{n} \omega_{k} p_{k}^{2}>\sum_{k=1}^{n} \omega_{k} p_{k}^{2} \geq p_{1}^{2 \omega_{1}} \cdots p_{n}^{2 \omega_{n}},
$$

by the AM-GM inequality for $p_{1}^{2}, \ldots, p_{n}^{2}$. Equality cannot be attained in this case.

Hence, we are left with the case $1 \leq m \leq n-1$. Define

$$
\begin{aligned}
& U=\omega_{1}+\cdots+\omega_{m}, \quad V=\omega_{m+1}+\cdots+\omega_{n}, \\
& A=\left(p_{1}^{\omega_{1}} \cdots p_{m}^{\omega_{m}}\right)^{1 / U} \text { and } B=\left(p_{m+1}^{\omega_{m+1}} \cdots p_{n}^{\omega_{n}}\right)^{1 / V} .
\end{aligned}
$$

Applying the weighted AM-GM inequality twice to the left hand side then yields

$$
\lambda \sum_{k=1}^{m} \omega_{k} p_{k}^{2}+\left(\sum_{k=m+1}^{n} \omega_{k} p_{k}\right)^{2} \geq \lambda \cdot U A^{2}+(V B)^{2} .
$$

On the other hand, using the same notation, the right hand side of (2.1) can be written as $p_{1}^{2 \omega_{1}} \cdots p_{n}^{2 \omega_{n}}=A^{2 U} B^{2 V}$, and hence we are left to prove that

$$
\lambda \cdot U A^{2}+(V B)^{2} \geq A^{2 U} B^{2 V} .
$$

Now, let $I=\left\{\sigma^{-1}(1), \ldots, \sigma^{-1}(m)\right\}$ in the original definition of $\lambda_{I}$. Then at this point in the proof (after rearranging our indices) we have $\sigma(I)=\{1,2, \ldots, m\}$. Hence, $\lambda_{\sigma(I)}=(1-U)^{-\frac{1-U}{U}}=f(U)$. Then, the maximality of $\lambda=f(\omega)$ implies

$$
\lambda \geq \lambda_{\sigma(I)}=(1-U)^{-\frac{1-U}{U}}=\left(\frac{1}{V}\right)^{\frac{V}{U}} .
$$

Finally, we can combine this with the weighted AM-GM inequality to deduce

$$
\begin{aligned}
\lambda \cdot U A^{2}+(V B)^{2} & \geq\left(\frac{1}{V}\right)^{V / U} \cdot U A^{2}+(V B)^{2} \\
& =U \cdot\left(\frac{A^{2}}{V^{V / U}}\right)+V \cdot\left(V B^{2}\right) \\
& \geq\left(\frac{A^{2}}{V^{V / U}}\right)^{U} \cdot\left(V B^{2}\right)^{V} \\
& =A^{2 U} B^{2 V}
\end{aligned}
$$

as claimed. This proves inequality (1.2).

Equality in the above occurs only if $\lambda=\lambda_{\sigma(I)}=\left(\frac{1}{V}\right)^{\frac{V}{U}}$ and $\lambda A^{2}=V B^{2}$. Filling in the definitions of $U$ and $V$, we see that $\lambda=\lambda_{\sigma(I)}$ implies that $\sigma(I)=\left\{i_{0}\right\}$ with $\omega_{i_{0}}=\omega$. Hence, this is exactly the claimed equality condition; this proves the 'only if' part. For the 'if' part, let $I=\left\{i_{0}\right\}$ and let $a, b$ be non-negative real numbers satisfying the given conditions. Denoting $u=\sum_{k \in I} \omega_{k}=\omega$ and $v=1-u=\sum_{k \notin I} \omega_{k}=1-\omega$, we have $\lambda=\lambda_{I}=v^{-v / u}$ and we have to show that 
$v^{-v / u} u a^{2}+v^{2} b^{2}=a^{2 u} b^{2 v}$, which is equivalent to $u\left(\frac{a^{2}}{v^{v / u}}\right)+v\left(v b^{2}\right)=a^{2 u} b^{2 v}$. Since we are given that $\lambda_{I} a^{2}=b^{2} \sum_{k \notin I} \omega_{k}$, we know that $\frac{a^{2}}{v^{v / u}}=b^{2} v$, yielding

$$
u\left(\frac{a^{2}}{v^{v / u}}\right)+v\left(v b^{2}\right)=v b^{2}=\left(v b^{2}\right)^{u} \cdot\left(v b^{2}\right)^{v}=\left(\frac{a^{2}}{v^{v / u}}\right)^{u} \cdot\left(v b^{2}\right)^{v}=a^{2 u} b^{2 v} .
$$

Hence the statement about the equality condition follows.

We have proven Theorem 2, Theorem 1 is now a straightforward corollary.

Proof of Theorem 1. For each integer $i$, with $1 \leq i \leq n$, we substitute $\left(a_{i}, b_{i}\right)$ by $\left(\left|a_{i}^{2}-b_{i}^{2}\right|, 2 a_{i} b_{i}\right)$ in inequality (1.2). Then inequality (1.2) in Theorem 2 reduces to inequality (1.1) in Theorem 1

Now we prove the propositions from Section 1

Proof of Proposition 3. We use the inequality $e^{t}>1+t$ for $t>0$ to deduce

$$
\lambda=(1-\omega)^{-\frac{1-\omega}{\omega}}=\left(\frac{1}{1-\omega}\right)^{\frac{1-\omega}{\omega}}=\left(1+\frac{\omega}{1-\omega}\right)^{\frac{1-\omega}{\omega}}<\left(e^{\frac{\omega}{1-\omega}}\right)^{\frac{1-\omega}{\omega}}=e,
$$

as claimed.

Proof of Proposition 4. Substituting $\omega_{1}=\ldots=\omega_{n}=\frac{1}{n}, b_{1}=a_{2}=\ldots=a_{n}=0$, $a_{1}=\left(1-\frac{1}{n}\right)^{\frac{n}{2}}$ and $b_{2}=\ldots=b_{n}=1$ in inequality (1.3) yields

$$
\mathcal{C}\left(1-\frac{1}{n}\right)^{n}+\left(\frac{n-1}{n}\right)^{2} \geq 1-\frac{1}{n}
$$

or equivalently,

$$
\mathcal{C} \geq\left(1+\frac{1}{n-1}\right)^{n-1}
$$

Taking the limit for $n \rightarrow+\infty$, we meet the desired estimation $\mathcal{C} \geq e$.

\section{ACKNOWLEDGMENTS}

We would like to thank Andrei Frimu for providing a translation of the article [1, Sungyoon Kim for detailed comments on an early draft of the paper, and the anonymous referee for several useful comments and suggestions.

\section{REFERENCES}

[1] V. Senderov and E. Turkevich, Problem M506, Kvant, 10(3) (1979), 35.

Department of Analysis, Loránd Eötvös University, Budapest, Hungary - and - Computer and Automation Research Institute (SZTAKI), Budapest, Hungary

E-mail address: kosgeza@sztaki.hu

Department of Mathematical Sciences, Seoul National University, Seoul, Republic OF KOREA

E-mail address: ultrametric@gmail.com

Department of Mathematics, Ghent University, B 9000 Gent, Belgium

E-mail address: Peter.Vandendriessche@UGent.be 